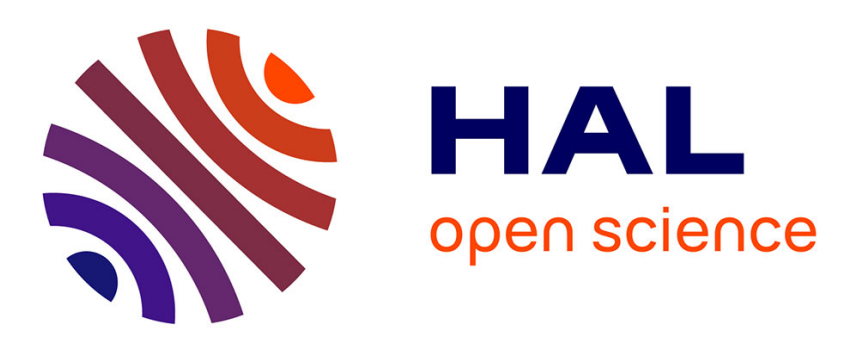

\title{
Accident climatique et fonctionnement de la société agricole [La sécheresse de 1976 chez les éleveurs d'un canton de la Nièvre]
}

\author{
Marie-Claire Robic, Françoise Plet, Violette Rey, Nicole Mathieu
}

\section{To cite this version:}

Marie-Claire Robic, Françoise Plet, Violette Rey, Nicole Mathieu. Accident climatique et fonctionnement de la société agricole [La sécheresse de 1976 chez les éleveurs d'un canton de la Nièvre]. Espace Géographique, 1982, 11 (2), pp.111-123. 10.3406/spgeo.1982.3726 . hal-02876669

\section{HAL Id: hal-02876669 https://hal.science/hal-02876669}

Submitted on 21 Jun 2020

HAL is a multi-disciplinary open access archive for the deposit and dissemination of scientific research documents, whether they are published or not. The documents may come from teaching and research institutions in France or abroad, or from public or private research centers.
L'archive ouverte pluridisciplinaire HAL, est destinée au dépôt et à la diffusion de documents scientifiques de niveau recherche, publiés ou non, émanant des établissements d'enseignement et de recherche français ou étrangers, des laboratoires publics ou privés. 


\author{
Marie-Claire Robic \\ Françoise Plet \\ Violette Rey \\ Mme Nicole Mathieu
}

\title{
Accident climatique et fonctionnement de la société agricole [ $\mathrm{La}$ sécheresse de 1976 chez les éleveurs d'un canton de la Nièvre]
}

\author{
In: Espace géographique. Tome 11 n², 1982. pp. 111-123.
}

\begin{abstract}
Résumé
La sécheresse 1976 a marqué l'agriculture française, et en particulier l'élevage, créant une véritable crise de subsistance pour les troupeaux. A travers le cas du canton de Luzy, cet aléa climatique unique est analysé comme un révélateur de l'état de l'agriculture et de la société agricole locale. Alors que les études sur le thème général des " natural hazards » évaluent les risques de catastrophe et l'ajustement des comportements aux risques encourus, notre examen de la sécheresse 1976 nous conduit surtout à repérer l'impact plus ou moins grand de la catastrophe sur les exploitations agricoles et les infrastructures rurales. Les réactions à l'événement comportent d'une part des initiatives collectives et des recherches de complémentarités nouvelles pour la survie des exploitations, d'autre part un fond important d'attitudes proprement individuelles, en même temps qu'une forte stabilité de certaines relations, telle la commercialisation, liée à la recherche de sécurité.
\end{abstract}

\section{Abstract}

Climatic accident and the agricultural community. The 1976 drought and the livestock farmers in a canton of Nièvre. - The 1976 drought has left its mark on French agriculture, and in particular on the raising of livestock, creating a real subsistence crisis for herds. Using the case of the Luzy canton, this unique climatic accident is analysed to reveal the state of agriculture and the local agricultural community. While studies on the general theme of natural hazards evaluate the risks of catastrophe and behavioural adjustments to the risks run, our examination of the 1976 drought above all leads us to register the greater or lesser impact of the catastrophe on farming and rural infrastructures. Reactions to the event consist on one hand of collective initiatives and the search for new ways to continue farming and on the other hand of a considerable substratum of actual individual attitudes, at the same time as a strong stability of certain relations such as commercialisation linked to the search for security.

Citer ce document / Cite this document :

Robic Marie-Claire, Plet Françoise, Rey Violette, Mathieu Nicole. Accident climatique et fonctionnement de la société agricole [La sécheresse de 1976 chez les éleveurs d'un canton de la Nièvre]. In: Espace géographique. Tome 11 n², 1982. pp. 111123.

doi : $10.3406 /$ spgeo.1982.3726

http://www.persee.fr/web/revues/home/prescript/article/spgeo_0046-2497_1982_num_11_2_3726 


\title{
ACCIDENT CLIMATIQUE ET FONCTIONNEMENT DE LA SOCIÉTÉ AGRICOLE
}

\author{
La sécheresse de 1976 chez les éleveurs d'un canton de la Nièvre
}

\author{
M. C. ROBIC, F. PLET, V. REY, N. MATHIEU \\ Equipe Analyse des espaces ruraux, L.A. 142, C.N.R.S., Paris-I
}

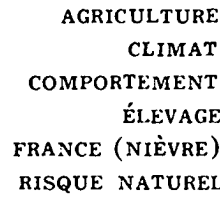

AGRICULTURE

BEHAVIOUR

CLIMATE

NIÈVRE, FRANCE

NATURAL HAZARD

STOCK BREEDINC

\begin{abstract}
RESUME. - La sécheresse 1976 a marqué l’agriculture française, et en particulier l'élevage, créant une véritable crise de subsistance pour les troupeaux. A travers le cas du canton de Luzy, cet aléa climatique unique est analysé comme un révélateur de l'état de l'agriculture et de la société agricole locale. Alors que les études sur le thème général des «natural hazards» évaluent les risques de catastrophe et l'ajustement des comportements aux risques encourus, notre examen de la sécheresse 1976 nous conduit surtout à repérer l'impact plus ou moins grand de la catastrophe sur les exploitations agricoles et les infrastructures rurales. Les réactions à l'événement comportent d'une part des initiatives collectives et des recherches de complémentarités nouvelles pour la survie des exploitations, d'autre part un fond important d'attitudes proprement individuelles, en même temps qu'une forte stabilité de certaines relations, telle la commercialisation, liée à la recherche de sécurité.
\end{abstract}

\begin{abstract}
Climatic accident and the agricultural community. The 1976 drought and the livestock farmers in a canton of Nièvre. - The 1976 drought has left its mark on French agriculture, and in particular on the raising of livestock, creating a real subsistence crisis for herds. Using the case of the Luzy canton, this unique climatic accident is analysed to reveal the state of agriculture and the local agricultural community. While studies on the general theme of natural hazards evaluate the risks of catastrophe and behavioural adjustments to the risks run, our examination of the 1976 drought above all leads us to register the greater or lesser impact of the catastrophe on farming and rural infrastructures. Reactions to the event consist on one hand of collective initiatives and the search for new ways to continue farming and on the other hand of a considerable substratum of actual individual attitudes, at the same time as a strong stability of certain relations such as commercialisation linked to the search for security.
\end{abstract}

\section{INTRODUCTION.}

\section{Problématique: la sécheresse comme accident.}

1976 reste dans les mémoires "lannée de la sécheresse ». Si les journaux de l'époque en gardent la trace. les analyses a posteriori sont peu fréquentes, et réductrices par rapport a l'événement. La plupart des études des géographes n'envisagent en effet la sécheresse de 1976 que comme phénomène naturel, à travers ses composantes climatologiques et à travers ses effets sur le milieu physique (sols, nappes, écoulements). Les autres types d'analyses ne semblent pas à la mesure de ce qui a été considéré, à chaud. comme un bienfait ou comme une calamité, en tout cas comme un événement exceptionnel. Ainsi. peu d'études se sont intéressées de près aux réactions des agriculteurs, au bilan économique de la sécheresse. aux enseignements à retenir de l'irruption d'un aléa climatique. 
Avec le recul, on peut certes comprendre cette discrétion. La sécheresse de 1976 n'a pas eu une dimension telle qu'elle puisse apparaître comme unc catastrophe irrémédiable, ni pour les exploitants pris individuellement, ni pour la production agricole, ni pour l'économic nationale. La sćchcresse est restée passagère: dès l'automne, la situation climatique est redevenue normale, et les années suivantes ont été plutôt pluvieuses. L'aridité n'a vraiment porté préjudice qu'aux agriculteurs, alors qu'une grande partie de la population a plutôt apprécié la canicule estivale. Même pour l'agriculture - y compris l'élevage, pourtant le plus touché - l'accident climatique a été surmonté avec une certaine facilité, grâce surtout à de multiples «expédients» internes au milieu agricole, qui ont montré la souplesse du système, sa capacité à intégrer l'aléa sans atteindre un point de rupture.

De plus, en raison même de son caractère inattendu, le phénomène était à saisir sur le vif, et pas seulement par des bilans a posteriori. Il fallait donc une disponibilité, une attention à l'événement peu compatibles avec des programmes en cours ou avec les démarches habituclles de la recherche. Il est vrai qu'au moment de l'enquête, les études sur le thème général des catastrophes naturelles étaient relativement peu connues en France. Ces recherches déjà anciennes se sont considérablement développées dans les pays anglo-saxons, au cours des années 1970 , sous l'expression de «natural hazards》(1). Elles sont axées principalement non pas sur les réactions à un sinistre effectif, ce qui constitue notre propos, mais sur l'appréciation de la probabilité d'occurrence d'une catastrophe, sur l'appréciation des dommages encourus et sur l'ajustement des comportements au risque. Ces préoccupations se traduisent par des investigations du type des enquêtes d'opinions. plus que par des analyses «immédiates» au moment de l'événement ou aussitôt après. Quelques recherches francaises (plutôt menées dans une optique d'aménagement) traitent de la réaction à des agressions extéricures, qu'elles soient d'origine strictement naturelle ou sociale (implantation d'une centrale nucléaire, marée noire...).

Notre recherche a reposé sur l'hypothèse que l'événement exceptionnel pouvait être un moment privilégié pour l'observation et la compréhension d'un espace et d'une société agricoles. Si du moins la crise qu'il provoque n'atteint pas l'ampleur d'une véritable catastrophe, cet événement exceptionnel peut être considéré come un révélateur. Il est un révélateur directement, pour l'observateur qui découvre des phénomènes mal perçus habituellement, et surtout pour les acteurs et à travers eux. Ceux-ci prennent en effet conscience de modes de fonctionnement réels. individucls ou collectifs, masqués dans une pratique quotidienne que laléa met en cause.

(1) Elles constituent d'ailleurs l'un des thèmes privilégiés de la commission "Homme et Environnement» de l'Union Géographique Internationale.
Sous la contrainte de la perturbation, ils sont obligés de mettre en question cette pratique, ce qui les amène à une vision nouvelle de leur histoirc, de leur présent et de leurs projets et peut-être à des attitudes nouvelles, plus ou moins durables.

\section{Un pays herbager: le canton de Luzy (Nièvre).}

Notre enquête a porté sur un espace où plusieurs d'entre nous avaient déjà travaillé (2), condition indispensable pour situer la forme et l'ampleur de la perturbation et pour permettre d'articuler sans trop de risques d'erreurs les apports des informations saisies sur le terrain. Les méthodes d'investigation, à base d'interviews, ont cherché à rendre compte de l'aspect exceptionnel de la sécheresse, à travers les récits et les analyses d'agriculteur's, de cadres d'organismes professionnels, de notables plus ou moins impliqués dans la production agricole.

Vieux pays herbager qui a été consacré à l'élevage par l'initiative de ses grands propriétaires terriens du siècle dernier, le canton de Luzy est un espace à dominante agricole, relativement homogène dans ses milieux naturels et ses structures d'exploitation. Un modèle agricole $y$ prévaut: le système peu intensif de production de bovins maigres (charolais), fondé sur l'herbe plus que sur les cultures fourragères. L'évolution des trente dernières années a été marquée par une augmentation de la charge bovine par unité de surface, accompagnée d'une substitution de lherbe aux labours. En outre les exploitations disposent d'assez «bonnes structures》, si l'on considère le rapport entre superficies, effectifs de travailleurs et tailles des troupeaux. La superficie moyenne des exploitations de plus de 5 ha s'élève à 45 ha (RGA * 1970): les unités de plus de 50 ha contrôlent 67 "; de la SAU *. Chaque actif agricole à temps complet dispose en moyenne d'une vingtaine dhectares. Le troupeau moyen est de l'ordre de 40 bovins, dont les trois quarts ont plus d'un an. Ces «bonnes structures» moyennes s'articulent sur une situation foncière marquée de longue date par la dualité entre château avec métairies ou fermes d'une part, petites unités familiales peu nombreuses d'autre part. et par la prééminence des faire-valoir indirects. Los rapports sociaux qui en résultent s'expriment à la fois par la persistance des liens interpersonnels, parfois accompagnés de relations de clien-

(2) Cf. BoNtrox J.C. (1976), "Transformations et permanences des pouvoirs dans une société rurale, à propos du sud Morvan ». Etudes Rurales, n" 63-64, p. 141-151. Boxtrox J.C.. Mathee N. (1977), La décitalisation de l'espace rural. lexemple de trois cantons du Morcan Sud. Paris, SEGESA-ACEAR. 69 p. + annexes. L'enquête a eu lieu clébut septembre. Référence globale: Boxwamour J. (1966). Le Morvan. la terre et les hommes. Paris; PUF. 454 p. - *RGA: Recensement Général de l'Agriculture; SAU: surface agricole utile; UGB: unité de gros bovins. 
tèle, et par la tradition d'une attitude politique de gauche.

Trois types d'exploitants se répartissent inégalement à travers le canton. Les gros exploitants, sur des unités supérieures à 100 ha, sont propriétaires, et plusieurs sont issus de familles nobles; aidés de salariés, ils opèrent dans un système d'exploitation relativement extensif à chargement en bétail réduit ( 0.9 à $1,3 \mathrm{UGB}^{*} / \mathrm{ha}$ ). Les exploitants moyens ont des superficies qui varient entre 50 et 100 ha avec une surface toujours en herbe très importante $(85 \%)$ et les plus forts chargements en bétail (1,7 à 2 UGB/ ha): leur intensification se traduit par un allongement du cycle et une augmentation du nombre des vaches-mères. Quant aux exploitants des petites unités, ils n'ont qu'un chargement modéré en bovins; c'est par la variété des élevages associés et les cultures complémentaires pour l'alimentation animale qu'ils pallient les inconvénients des faibles surfaces. Au total, le système adopté par la grande majorité des agriculteurs exige une forte immobilisation de capitaux; il comporte une certaine vulnérabilité, liée à l'augmentation du chargement, à l'abandon des cultures et à la spécialisation des exploitations vers une quasi-monoproduction de viande, laquelle exclut des complémentarités entre différentes productions.

C'est donc dans cet espace, sans problème particulier par rapport à d'autres régions d'élevage à viande, que nous avons abordé l'étude de ce «risque naturel» que fut la sécheresse de 1976. Après en avoir rappelé le vécu et la mesure, nous avons analysé les effets de l'aléa sur le fonctionnement des exploitations et des équipements et identifié les comportements individucls et collectifs qu'il a suscités. A chaque niveau de l'analyse, il apparaît que des décalages de perception dans lintensité du phénomène ou dans les étapes de son déroulement régissent en grande partic les attitudes.

\section{L'ANNÉE 1976 A LUZY : UNE SÉCHERESSE EXCEPTIONNELLE.}

La sécheresse a été durement vécue par les agriculteurs et, le temps d'une saison. a marqué les récits, les réflexions et parfois les confidences. Plus directement que des mesures physiques. le langage employé alor's exprimait l'ampleur de l'événement et en restituait le vécu quotidien.

\section{Chronique du vécu.}

... Ici la sécheresse a été catastrophique: le canton de Lury est un des plus secs de la Nièvre $\gg .$.
«La sécheresse de 1911 avait été moins longue ». ... Quant à la sécheresse célèbre de 1893, bien sûr «on mettait des draps sur les charrettes pour empêcher le foin de passer entre les claies, mais on avait de la paille à donner à manger aux bêtes et puis on était autorisé à mener les bêtes dans les bois du château »...

Les appréciations relatives au temps du printemps restaient pourtant diverses : «A Millay, il n'avait pas plu depuis le 8 février, à Flety non plus, à part une ondée en mars». "A la mise au pré, en marsavril, les prés étaient secs, ça manquait d'eau partout ». D'autres agriculteurs estimaient au contraire «qu'il y avait eu un beau printemps; en avril ça allait, les réserves d'eau étaient habituelles». Certains «ont cru à la pluie jusqu'à fin mai ». De telles évaluations s'accompagnaient de jugements amers sur le «gaspillage de l'herbe généreuse du printemps».

La période des foins fut très difficile, la pousse de l'herbe restant très faible: «ça grainait à 15$20 \mathrm{~cm}$ sans monter ». La récolte de foin qui représente en temps normal l'essentiel du fourrage pour l'hiver fut très maigre: «on comptait les bottes et non les remorques». Certains durent enclore les prés de fauche pour les faire pâturer et la première coupe n'atteignit souvent que le «tiers 》 de la récolte habituelle: «ici, on avait eu déjà des sécheresses après les foins, mais jamais pour la première coupe, on était novice».

Début juillet commençait la sécheresse chaude, c'était la «canicule»; au cimetière, la terre était sèche à $1,40 \mathrm{~m}$ de profondeur. La course aux approvisionnements pour pallier la faim du bétail domine alors la vie locale. "Quel travail ça a été la paille! Aujourd'hui on ne peut plus se rendre compte de l'état des prairies: fin juillet c'était le Sahara, là, dans le pré en face. Les bêtes se sauvaient, elles ont mangé les haies. La coopérative a été dévalisée de clôtures à remplacer». Courant août, quelques orages dans la partie orientale et pré-morvandelle $\mathrm{du}$ canton eurent pour effet de raviner certains champs en pente. Début septembre seulement arrivèrent «les premières pluies sérieuses» dont on espérait qu'elles feraient repartir les pâtures et pousser le seigle. les vesces et les choux fourragers, exceptionnellement semés pour faire un fourrage de complément pour l'hiver.

Toutefois le pessimisme sur le devenir des prairies demeurait une attitude quasi générale: «les vieilles pâtures seraient plus ou moins perdues» car «la couenne des prairies (la couche superficielle du sol où s'enchevêtrent des racines) ne repartirait pas ».

Ces fragments, brefs témoignages du contenu des récits, sont le reflet de la perception dramatique que la majorité des agriculteurs ont eue de l'accident. L'abondance de la terminologie et la précision des expressions soulignent l'acuité de l'observation des phénomènes physiques et économiques. La fréquence 
des références au passé suggère le besoin de repères face à une catastrophe afin, semble-t-il, d'en relativiser la portée.

\section{Conjonction exceptionnelle de deux effets climatiques.}

La mesure physique de la sécheresse, évaluée à travers les relevés météorologiques, confirme l'ampleur et la durée du phénomène. Elle permet en outre de mieux cerner certains décalages entre les épisodes et la perception de leurs conséquences.

Par rappor't à la sécheresse qui a sévi sur l'ensemble de la France, celle des confins nivernaismorvandiaux se caractérise par une succession marquée des deux phases saisonnières du phénomène. En effet, pendant l'hiver 75-76, alternent dans la Nièvre mois plus humides que la moyenne (septembre, novembre) et mois plus secs (octobre, décembre, janvier) (fig. 1); sans qu il y ait rien encore d'alarmant, le déficit est déjà sensible et rattache la Nièvre à l'aire de sécheresse hivernale d'origine continentale qui a affecté l'enscmble BourgogneChampagne-Franche-Comté. Pendant le printemps et l'été, la zone nivernaise se rattache alors à l'aire de sécheresse des provinces atlantiques dont un golfe s'avance jusqu'en Bresse (fig. 2).

L'observation est identique en ce qui concerne les températures. Le froid du printemps 1976 est particulièrement marqué dans la France de l'Est; des gelées tardives renforcent la sécheresse précoce et retardent le démarrage de la végétation, tandis que le vent desséchant du NE, habituel au mois de mars, se maintient jusqu'en juin. A partir de juin, la diagonale des fortes chaleurs qui traverse le territoire national, des Charentes à la Champagne, englobe la Bourgogne et la Bresse.

Le caractère exceptionnel de la sécheresse dans la Nièvre apparaît d'autant mieux quand on le compare aux volumes de précipitations des autres grandes sécheresses: à Nevers, entre le $1^{\text {"r }}$ avril et le 31 août 1976, il n'est tombé que $99 \mathrm{~mm}$ de pluies, contre 188 en 1893, 222 en 1906, 278 en 1921, 228 en 1949. Ce diagnostic sur la sécheresse en Nivernais montre combien cette région a été parmi les plus touchées de la France continentale, parce qu'alternativement rattachée à la France de l'Est et à la France de l'Ouest, dont elle a cumulé les handicaps en 1975 et 1976 .

Au total, la perception dramatique, comme la mesure de l'exception, convergent pour éclairer la totale impréparation, la totale imprévoyance des producteurs devant ce qui reste un «natural hazard», sinon une catastrophe pour la région. D'ailleurs les agriculteurs, pour expliquer leur oubli de cet aléa et leur négligence dans la constitution de stocks, ont suuligné que leur région n'avait pas connu, dans les années précédentes, les légères irrégularités pluvio- métriques enregistrées dans d'autres zones (France de l'Ouest) (3).

\section{EXPLOITATIONS AGRICOLES ET ÉQUIPEMENTS RURAUX FACE A L'ACCIDENT.}

Par sa brutalité, la sécheresse a frappé de plein fouet les exploitations agricoles et a altéré leur fonctionnement en accusant leurs différences et leurs disparités par un surcroît de travail et de dépenses. Dans le même temps, elle révélait l'importance et le rôle de certains éléments de l'infrastructure rurale et de l'organisation para-agricole, ignorés ou minimisés dans le fonctionnement de l'agriculture en temps normal; plus, elle amorçait même certaines de leurs transformations.

\section{Perturbation dans les exploitations et disparités nouvelles.}

Au terme des enquêtes, deux impressions contradictoires paraissaient l'emporter : d'une part la brutale égalité de tous devant les prés d'août transformés en «paillassons», de l'autre une quasi irréductible diversité et variabilité des atteintes de la sécheresse selon les exploitants et les exploitations. Néanmoins, on peut considérer que trois sortes de facteurs sont inégalement intervenus, ont accentué les différences entre les exploitations et ont fait apparaitre des disparités nouvelles, temporaires ou durables. A la dimension des unités, s'ajoutent des éléments liés à leur structure spatiale et d'autres propres à l'état de la cellule familiale.

La localisation et la position topographique des parcelles ont introduit ou renforcé les disparités liées à la répartition spatiale des terres des exploitations et à la position de chacune d'elles dans l'espace étudié. Les pâtures de haut de versant, où les sols sont les plus minces, ont été les plus abîmées par le piétinement. La présence ou non de sources, la pérennité plus ou moins grande des nappes ont également joué; dans certains cas le tarissement a été total, dans d'autres il y a eu changement de place sur le versant, ce qui a nécessité de recreuser les mares; au contraire, dans la dépression de Luzy, les puits se rechargeaient la nuit, permettant presque en permanence un approvisionnement naturel; enfin les prés de fond de vallée, jamais fauchés par excès d'humidité en année normale, ont pu fournir une coupe de foin. L'équipement en adduction d'eau, selon les communes, la distance des exploitations et des parcelles par rapport au réseau, ont été un autre générateur d'inégalités : toutes les exploitations

(3) $1972: 800 \mathrm{~mm}$, mais année fraiche; $1973: 817 \mathrm{~mm}$ avec été marqué; $1974: 1015 \mathrm{~mm}$ avec exceptionnel étalement des pluies; $1975: 898 \mathrm{~mm}$ à Luzy. 
Fig. 1. - Précipitations à Luzy. L'étoile indique la hauteur moyenne mensuelle en $\mathrm{mm}$ pour la période $1931-$ 1960; le total annuel moyen a été de $860 \mathrm{~mm}$. ta $=$ total annuel.

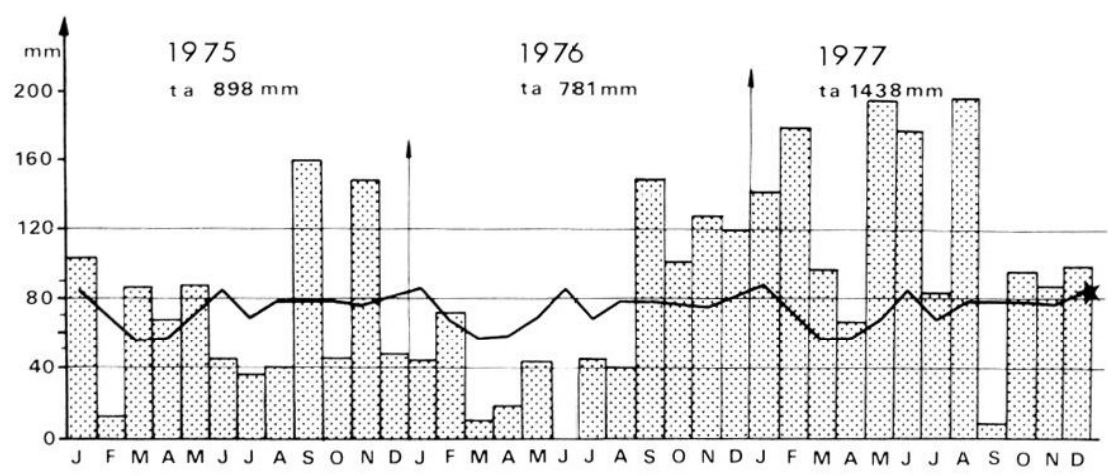

ont manqué de fourrage, partout les bêtes ont eu faim, mais toutes n'ont pas manqué d'eau. L'anomalie climatique a dévoilé d'autres différences devant le milieu physique, rendant plus dure la position des exploitants qui n'étaicnt pas desservis, surtout à l'ouest où des sols sableux les privaient de toute solution de rechange; au contraire, à l'est, quelques orages le long de la vallée de l'Arroux ont amélioré l'état de certaines prairies.

Les caractéristiques et la composition de la cellule familiale, les fonctions de certains de ses membres, ont permis ou non de mieux faire face aux contraintes imposées par la sécheresse. Ainsi, être membre du conseil d'administration du Crédit Agricole ou président de la coopérative a pu conférer certaines facilités, parmi lesquelles un meilleur accès au crédit. La multi-activité a donné à certains exploitants (rabatteur, régisseur, exploitant marchand de bestiaux) des opportunités commerciales pour la vente des animaux ou pour l'achat de nourriture. Il faut également souligner l'importance des différentes formes des rapports entre famille et exploitation, et de la participation des membres de la famille vivant sur l'exploitation au surcroît de travail. En dehors de grandes unités, pour lesquelles exploitation et famille sont radicalement séparées, de grandes inégalités se sont révélées. Aux exploitations tenues par des célibataires ou des ménages avec enfants en bas âge, n'ayant qu'une force de travail limitée et inélastique, s'opposent les exploitations mieux dotées (deux ménages - pèréfils - ou couples avec enfants d'âge scolaire) qui ont pu mobiliser des bras supplémentaires pour aider aux charrois, à l'alimentation, aux cueillettes. De même, la présence ou l'absence de ressources extra-agricoles apportées par l'un des membres de la famille (salaires, retraites) a introduit un clivage financier entre des familles peu ou fortement touchées. Le profil psychologique particulier à chaque exploitant a également joué, orientant la réaction vers le laisseraller. la dépense ou la résistance désespérée. Enfin certaines coïncidences se sont parfois greffées, modifiant linfluence du phénomène: ainsi en fut-il d'un vieux stock de paille et de foin, ou de l'accouchement d'une agricultrice en août.

Au-delá de l'étonnante variabilité des situations individuelles qui ont modulé l'intensité de l'agres-

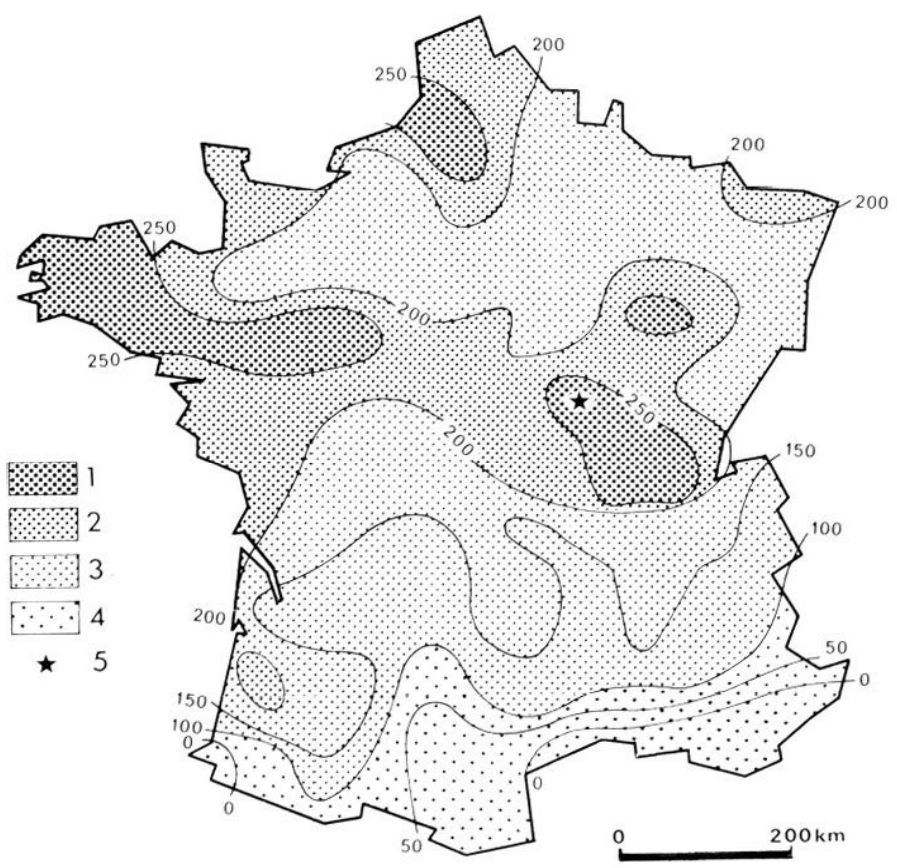

FIG. 2. - Déficience des précipitations agricoles utiles. Ecart 1976 - année moyenne. Source: Lecarpentier (1976). Ce bilan est calculé de la manière suivante:

$$
\frac{\mathrm{ETP}-(P+\mathrm{RU})}{1976}-\frac{\mathrm{ETP}-(\mathrm{P}+\mathrm{RU})}{\text { année moyenne }}
$$

ETP -. évapotranspiration potenticlle

$P=$ précipitations

$R U$ =- réserves d'eau facilement utilisée, constante évaluée à $50 \mathrm{~mm}$

1. Plus de 250. - 2. 200 à 250 - 3. 100 à 200. - 4. Moins de 100. - 5. Canton de Luzy.

sion de la sécheresse sur les exploitations, la dimension économique demeure un critère prépondérant. Une première illustration en était fournie dès septembre par l'etat des herbages: dans les exploitations à système peu intensif et à faible charge en bétail. les pâtures étaient moins abimées et le redémarrage fut plus facile. Le dactyle, graminée aux racines profondes, émcrgeait par sa meilleure résistance: malheureusement son coût élevé (engrais, rotation rapide pour éviter le «feutrage au sol ») 
semblait le réserver aux seules exploitations de pointe. Malgré l'impossibilité d'établir une échelle stricte des dommages, il apparaît que les pctites et surtout les moyennes exploitations ont subi le plus de préjudices. C'est dans ces groupes, fidèles au système régional dominant d'élevage de bêtes maigres, que la tendance à l'augmentation de la charge du cheptel de souche a été la plus nette; or elle s'est réalisée le plus souvent dans le cadre d'une force de travail restée stable et au prix d'un endettement. Pour eux, la résistance à la sécheresse ne pouvait guère passer que par l'augmentation de l'endettement, la décapitalisation par des ventes d'animaux reproducteurs (vaches et brebis) et le différé des investissements prévus (matériel, stabulation). Parmi eux les plus touchés furent ceux qui, jeunes et n'ayant que de petites superficies, s'étaient engagés dans un processus de croissance cumulant l'augmentation du cheptel et de la taille au prix d'un fort endettement; la crise les a mis dans une situation très critique, s'est répercutée sur le niveau de vie de leur famille et a entamé leur épargne. Par contre, les exploitations les moins touchées furent les grandes exploitations aux systèmes largement extensifs et celles ayant des élevages originaux. Au prix de quelques retouches dans leurs pratiques habituelles, à l'aise dans leurs trésoreries ou dans leur accès au crédit, "aristocrates de l'herbe», ils ont bien résisté. Enfin, parmi les petites unités habituellement caractérisées par des pratiques agricoles conservant les cultures fourragères, la crise de la sécheresse s'est traduite par l'utilisation maximale de toute la main-d'ouvre familiale, par la multiplication des cultures dérobées assurant une autonomie de l'alimentation du bétail grâce à des ressources plus diversifiées. Si l'orientation du système d'exploitation n'a pas été atteinte comme dans le cas des exploitations de la catégorie intermédiaire, le niveau de vie familial a été fortement réduit.

\section{Un rôle nouveau pour les infrastructures de service?}

Le réseau d'adduction d'eau, le chemin de fer et la coopérative comptent parmi les équipements ruraux qui ont été les plus sollicités lors de la sécheresse, et dont le rôle s'est trouvé modifié.

\section{a) Le réseau d'eau.}

En ce qui concerne les infrastructures d'approvisionnement en eau, la politique appliquée dans le canton depuis les années 1950 est relativement typique du modèle moyen en milieu rural. La solution aux problèmes des captages et des réseaux de distribution a été confiée à un syndicat intercommunal chargé de mettre en place un réseau collectif. Destiné à apporter en priorité un élément nécessaire de confort plus qu'un outil d'intensification agricole, il est d'autant plus onéreux (et d'implantation tardive) que l'habitat est dispersé. C'est pourquoi le taux de desserte des localités par les réseaux de distribution est tributaire du degré de dispersion de l'habitat.

La sécheresse a d'abord révélé les insuffisances du réseau: il s'est avéré inapte à assurer l'ensemble des besoins en eau, en particulier pour l'usage agricole. Les réservoirs étaient vides certains jours, des villages se sont trouvés isolés faute d'interconnexion entre les réseaux, et ailleurs les débits manquaient pour permettre de pomper l'eau d'une station à une autre.

Comme nous l'avons précédemment montré, l'accident climatique a fait ressortir les inégalités devant cet équipement collectif. Il a renforcé deux tendances contradictoires par rapport à la politique de l'eau. En modifiant la perception du besoin en eau, il a provoqué une prise de conscience de la nécessité d'un système de gestion collective d'une ressource, l'eau, et donc du renforcement du réseau, de la généralisation de l'interconnexion, de l'amélioration et de la multiplication des captages, même après le retour à la normale. Et ceci d'autant plus que l'augmentation des besoins en eau pour l'élevage avait jusque-là été négligée dans l'évaluation de l'équipement en eau.

La sécheresse a également renforcé les convictions de ceux qui préconisent l'autonomie des collectivités locales ou des exploitations. Certaines communes ont recherché une solution locale à l'approvisionnement en eau; beaucoup d'exploitations ont cherché à améliorer la connaissance et la surveillance des sources, tout en remettant ou mettant en service des pompages individuels sur leurs puits. La sécheresse aura été l'occasion, d'une manière générale, d'améliorer les captages et l'entretien des sources. La sécheresse a donc révélé la timidité des conceptions primitives et la mise au second plan de la question de l'approvisionnement en eau de l'agriculture par rapport à d'autres priorités; elle a aussi posé le problème de l'affermage du réseau à une société privée (la Lyonnaise des Eaux) qui s'avère être un instrument de sclérose, même s'il a l'avantage, pour les collectivités locales, de simplifier la gestion. C'est donc l'ensemble de la politique locale de l'eau en milieu rural que la sécheresse a mise en question, sans qu'un choix clair se manifeste entre la délégation de la gestion des ressources en eau ou la recherche de systèmes autonomes.

b) Le chemin de fer.

Les répercussions sur la gare de Luzy ont été d'autre nature. Sur une voie ferrée secondaire type d'infrastructure apparemment condamné actuellement - elle ne doit vraisemblablement son maintien en activité qu'à la présence, sur la ligne, de centres industriels (Decize, Cercy-la-Tour), en même temps qu'à la persistance d'un fief de gauche s'oppo- 
sant à la suppression des services publics. En temps normal, la gare de Luzy ne s'anime qu'aux heures de retour scolaire et aux vacances. L'aléa a fait redécouvrir son importance. Elle a été le point de départ des wagons chargés de tracteurs se rendant au «chanticr de paille» de Pont-sur-Yonne (voir infra) et le point central de réception des pailles et foins récoltés ou achetés hor's de la région. La réception de la récolte de Pont-sur-Yonne s'est étalée sur un mois et demi au rythme d'un train quotidien de 3 wagons de paille. Une place de gare conçue à l'échelle de sa fonction ancienne de gros marché agricole et de sa fonction de chef-lieu de canton, l'existence, dans son prolongement, de l'immense foirail avec sa bascule, en ont fait le point de convergence de tous les tracteurs de la région venus chercher leur livraison. Animation certes éphémère, mais qui a marqué suffisamment les esprits pour que les municipalités du canton voient d'un très mauvais ceil tout projet de restriction concernant la circulation des trains et le nombre des arrêts. La lutte contre la sécheresse a rappelé ou renforcé la conscience des risques graves liés à l'isolement rural, et de l'importance du service public qui relie.

\section{c) La coopérative.}

Dans la capacité d'intégrer collectivement et régionalement l'aléa, la coopérative agricole de Luzy a eu un rôle déterminant. Cette coopérative représente la forme mutualiste «minimum» compatible avec le système d'élevage charolais dominant. Elle assure la collecte des céréales d'une région qui en fait peu (35 à $40 \%$ de son chiffre d'affaires) et facilite l'approvisionnement de ses 300 à 500 utilisateurs réguliers à un moindre prix pour les besoins de l'élevage (engrais, clôtures, matériel pour l'élevage, compléments, etc.). Elle a modérément développé, avant 1976, un service de fabrication d'aliments du bétail dans le prolongement de ces deux activités. Comme, d'ailleurs, le petit abattoir de Luzy spécialisé dans les bêtes accidentées, la coopérative a joué le rôle d'un organisme tampon, ou d'un système de recours retrouvé face à la calamité, réponse d'une organisation mutuclle à la crise vécue par ses adhérents et, au-delà, par l'ensemble des éleveurs de la région. Elle a été un instrument clé pour l'approvisionnement des exploitants du canton en organisant le plus gros chantier de ramassage de paille (2500 tonnes récoltées à Pont-sur-Yonne sur 1200 ha) et en se constituant le plus gros vendeur de paille de la région (1850 t en août et septembre 1976,2680 t d'août 76 à mars 77). Son activité pendant l'été 76 et dans la période suivante enregistra en écho les modifications immédiates ou décalées du système agricole entraînées par la sécheresse. La baisse des postes de collecte de céréales et de vente d'engrais reflète, dans la comptabilité de la coopérative, les difficultés auxquelles se heurtent les agriculteurs. Le chiffre d'affaires s'est trouvé, par contre, gonflé par les achats «anormaux» pour l'alimentation bovine, d'abord la paille, puis le foin de la Crau et les céréales. Le volume de fabrications et de ventes d'aliments composés a été exceptionnel durant l'été, puis l'hiver, car les aliments ont assuré la soudure et permis aux bîtes de regagner du poids pour le printemps suivant.

Elle a eu incontestablement un rôle de régulateur économique, et a été un recours majeur dans la crise, par sa capacité de réponse aux demandes des éleveurs. Elle a également aidé ses adhérents à supporter les dificicultés de trésorerie en accordant des facilités de paiement et en obtenant du Crédit Agricole un «prêt de préfinancement calamité sécheresse ». Elle a enfin contribué à régulariser les prix, puisquen définitive le prix de la paille pratiqué à la coopérative a été inférieur au prix moyen du négoce, et que progressivement les éleveurs se sont tournés pour leurs achats vers la coopérative, qui a dès lors pris une place prépondérante dans le marché des fourrages.

Ainsi, dans la conjoncture exceptionnelle, la coopérative a montré sa souplesse potentielle et sa capacité d'amortir une crise pour la région toute entière. Mais s'agit-il d'une réponse éphémère, aussi éphémère que l'a été la calamité elle-même? Le gros effort de fabrication, la création de nouveaux réseaux d'approvisionnement et de clientèle ont permis à la coopérative de se faire une place de choix dans le secteur des aliments composés, encore peu utilisés dans ce système de production. Ce changement d'orientation dans le fonctionnement de la coopérative indique sans duule l'amorce d'un changement dans la conduite alimentaire de l'élevage régional.

\section{COMPORTEMENTS INDIVIDUELS ET COLLECTIFS DANS LA CRISE.}

L'analyse des comportements manifestés pendant la sécheresse n'a pas manqué de mettre à jour des contradictions. L'appreciation de ces comportements a parfois été délicate, tant s'imbriquent dans les interviews les expressions de défense collective de la profession («on »). daffirmation de sa propre identité ( je»), assortie de la dissociation d'avec «eux», «les autres».

Plusieurs «pistes" s'ouvraient au départ de l'enquête, dans une interrogation d'ensemble sur les modes de réaction à la crise. Avait-on apporté des innovations dans les pratiques spatiales et sociales? Sur quelles trames de relations s'étaient-elles dessinées? Les réactions étaient-elles à base individuelle ou collective? Avaient-elles suscité des organismes particuliers ou reposaient-elles surtout sur des "institutions pré-existantes? Au total, face a l'agression constituée par la sécheresse et face 
aux risques encore difficiles à évaluer au début septembre, quelle était la gamme des attitudes? Quelle a été la dynamique temporelle de ces comportements? Y a-t-il eu solidarité ou division, individualisme ou comportements collectifs, homogénéité ou hétérogénéité? Retrouvait-on, réactivés plus qu'étouffés, les clivages socio-politiques antérieurs ?

Au départ, deux informations: l'une, émanant de la Direction Départementale de l'Agriculture, soulignait la mise sur pied d'un «Comité paille», remarquable témoignage de solidarité et d'organisation entre agriculteurs; l'autre, issue plutôt de notables ou de "para-agricoles», faisait état d'un climat de panique. Qu'en était-il réellement ? La crise étaitelle si forte qu'elle suscitât des réactions extrêmes, du moins exceptionnelles, d'affolement ou de nouvelles solidarités? Au contraire, les premiers contacts nous ont confrontés à une juxtaposition de solutions particulières et pondérées, où les inventions, nombreuses, se sont largement passées de réseaux et d'organismes créés de toutes pièces.

\section{Y a-t-il eu "panique"?}

L'image d'un «climat de panique» s'est effacée rapidement au cours de l'enquête. Les réactions d'affolement, dénoncées en termes d'achats inconsidérés de paille, de foin, de clôtures, d' "alimentation irrationnelle», de «ventes prématurées», n'ont pas eu l'ampleur que leur conféraient les discours de notables (vétérinaires, marchands de bestiaux, élus locaux...). Les manifestations de l'inquiétude des éleveurs ne se sont pas produites simultanément et elles n'ont pas pris le tour précipité et excessif qu'on leur prêtait. Seule une minorité reconnaît avoir agi à la hâte en achetant de la paille, trop cher, début juin, ou en vendant à perte du bétail pour acheter des aliments. Cette estimation peut ëtre sujette à caution dans la mesure où il paraît naturel de ne pas reconnaître ses propres erreurs. De fait, le contrôle par la date de vente des animaux et par la nature de ces ventes a permis de repérer des transactions "anormales» parce qu'anticipées, voire non prévues dans le plan de développement, ou par rapport à une année moyenne.

En tout état de cause, ces comportements de vente ont moins traduit une attitude inconséquente que la perception d'une situation critique, la conscience d'un risque de déséquilibre momentané, dont la prévention devait passer éventuellement par le s enoncement à un certain plan de développement. Ils sont l'expression d'une décision raisonnée face à la découverte de la fragilité d'un système de production à spécialisation et intensification trop rapides et de la remise en cause du bien-fondé des choix économiques antérieurs. Dans cette appréciation de la situation critique, beaucoup d'éléments, relatifs à la situation de l'unité de production, à la situation familiale momentanée, aux traits de per- sonnalité de l'agriculteur et à son rapport au travail, sont intervenus. Les manifestations de «panique» ont été plus nettes chez les exploitants ayant une trop forte charge de bétail (1,3 bovin à l'hectare et plus), mais elles sont restées exceptionnelles. En fait on se trouvait en présence d'une succession temporelle : une cascade d'événements étalés a pu donner l'illusion d'une propagation de comportements irrationnels. Les notables, non directement impliqués dans la crise, l'ont alors taxé de panique sans qu'on puisse toutefois en conclure à un tel phénomène. Il n'y a eu qu'une collection de décisions individuelles obéissant à des logiques diverses et à des situations critiques échelonnées.

\section{Y a-t-il eu réaction solidaire?}

Une attention particulière a été portée au «Comité paille», car il a été présenté de l'extérieur comme une réponse collective et solidaire des «agriculteurs de Luzy ». A travers lui, c'était s'interroger sur l'émergence d'un nouveau «mutualisme» face à la catastrophe, et sur les formes spatiales que ce mutualisme pouvait susciter, formes nouvelles ou structures latentes (4). A son propos, les thèses sont d'emblée contradictoires. Pour certains, membres actifs du syndicat communal FDSEA (5) de Millay ou notables locaux, il s'agissait bien d'une forme collective spontanée, expression même de solidarité. Pour d'autres, ce fameux comité, télécommandé par la DDA, n'aurait été qu'une opération destinée à prouver l'efficacité du syndicat des «gros». Dans les faits, il a été organisé fin juin au cours d'une réunion d'information du syndicat communal FDSEA de Millay, où fut proposée la mise sur pied d'un chantier pour collecter la paille à Pont-sur-Yonne. Les agriculteurs volontaires (avec ou sans leur tracteur) devaient être rémunérés. Sous l'impulsion du syndicat de Millay, la coopérative s'est associée au projet et a organisé matériellement le chantier; elle y a détaché un de ses employés durant deux mois. Dès lors, l'action du comité est devenue difficile à dissocier de l'activité intense de la coopérative, et l'on peut se demander quelle aurait été l'efficacité du comité sans le relais de la coopérative et son soutien logistique.

La participation (ou la non participation) des agriculteurs au chantier et leurs motivations ont été un excellent révélateur des clivages économiques et politiques. L'obligation minimale de 5 jours de travail a eu un effet sélectif évident parmi les agriculteurs,

(4) Cf. les recherches sur les «pays» et particulièrement sur les «espaces de solidarité », éventuellement repérables quand «un processus d'identification où on nie provisoirement toutes les différences au profit de la ressemblance intervient en réponse à une agression» (UzAN, 1978).

(5) FDSEA : Fédération départementale des syndicats d'exploitants agricoles. - DDA: Direction départementale de l'agriculture. 
déjà soumis à un surcroît de travail provoqué dans chaque exploitation par la sécheresse. Seules les grosses unités, capables de se délester d'un homme et d'un tracteur, ont pu se permettre d'envoyer un de leurs actifs ou une partie du matériel sur le chantier; d'autre part, quelques exploitants petits ou moyens ayant une main-d'œuvre familiale abondante ont espéré valoriser ainsi leur force de travail et atténuer leurs problèmes de trésorerie.

Mais d'autres paysans, dès le 12 juillet, ont exprimé leur refus d'y travailler; pour les uns, la riposte collective était trop tardive par rapport à leurs initiatives individuelles; ou bien, paysans opposés à la Fédération et au syndicat majoritaire, ils dénonçaient une collusion entre le syndicalisme et les grands propriétaires fonciers suivis de leur clientèle de fermiers, et le montage d'une réponse de la «profession organisée» face à l'épreuve. L'éventail des acheteurs de paille à la coopérative a été plus ouvert. La coopérative a été considérée par les uns comme un marchand de paille comme les autres proposant un prix très compétitif; d'autres ont fait confiance à cet organisme, considéré comme la propriété des paysans et le prolongement de ses adhérents; d'autres enfin se sont crus obligés d'acheter de la paille à la coopérative, soit pour bénéficier des possibilités de crédit qu'elle offrait, soit par une sorte d'obligation morale liée à leurs fonctions dans le syndicalisme local.

C'est, au fond, une institution «normale» qui a assumé l'aspect collectif de l'approvisionnement en paille, ne laissant de l'initiative originelle qu'une controverse sur la pertinence de la création d'un organe nouveau.

\section{3. ... ou des innovations individuelles?}

Les clés d'interprétation qui nous étaient proposées: panique/solidarité, se sont ainsi peu à peu dissoutes. C'est l'image de solutions individuelles plutôt que collectives qui s'est imposée, et par là d'une très grande diversité des réactions.

Ainsi, pour l'approvisionnement en paillc, la grande majoritć des agriculteurs a procédé individuellement ou par petits groupes. La nature de ces petits groupes est très variée: groupes syndicaux, groupes familiaux, groupes de voisinage (d'une même commune), groupes d'entraide habituelle (communes voisines), groupes de propriétaires-fermicrs, etc., se sont mobilisés pour aller chercher la paille. L'existence éventuelle de nouvelles pratiques locales de regroupement entre exploitants (constitution de groupes nouveaux, ruptures d'associations habituelles, renforcement des liens préexistants) ne s'est pas clairement imposée à travers les enquêtes. Ce qui a semblé net. c'est l'exceptionnelle activité de ces multiples groupes que l'on a vus à l'œuvre.

Liexplication de cette majorité d'actions individuelles et spontanées est triple.
1. Elle peut tenir à la passivité, au moins au début, des organes officiels: la coopérative n'a agi que tardivement et il ne s'est pas constitué dans toutes les communes des comités syndicaux (mais le taux de syndicalisatior est faible). La méfiance à l'égard des organisations «politico-syndicales», qu'elle soit d'origine personnelle ou expression d'une opposition politique, a aussi réduit l'audience de ces institutions; s'y ajoute enfin la concurrence entre organisations.

2. Elle peut révéler le désarroi, l'affolement d'agriculteurs qui, sans aller jusqu'à des actions inconsidérées (achats excessifs de paille, ventes de bétail anticipées) se sont néanmoins précipités pour agir, sans attendre la mise en route de l'action des organisations ou d'autres solutions collectives.

3. Ne révèle-t-elle pas surtout, plus que l'individualisme paysan, l'individualité fondamentale de chaque exploitant, qui s'exprime jusque dans son réseau de relations? D'ailleurs, ccux qui sc sont trouvés pris au dépourvu n'ont-ils pas eu mauvaise conscience d'avoir été mal préparés à faire front à une calamité naturelle (complexe de mauvais paysan) et n'ont-ils pas cherché à agir isolément

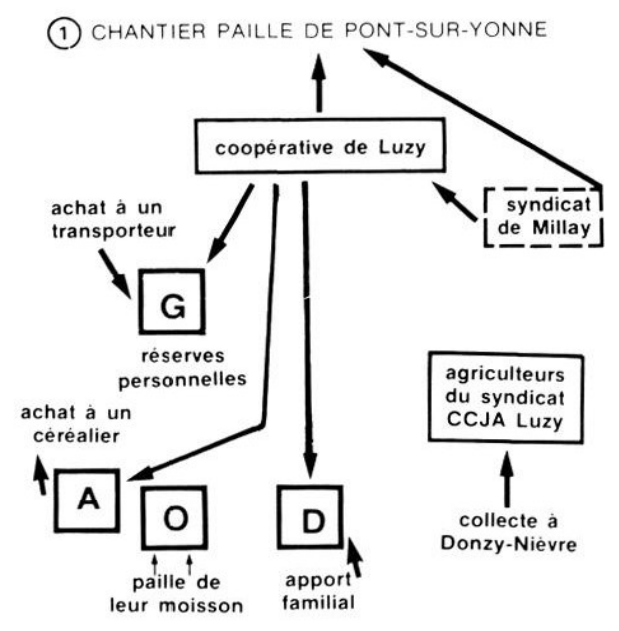

(2) AUTRES FORMES D'APPROVISIONNEMENT

EN GROUPE

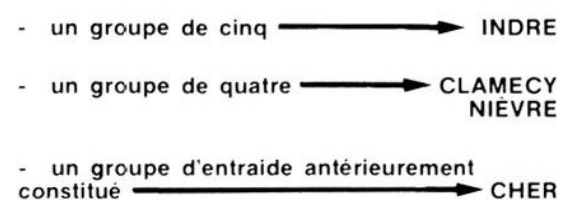

INDIVIDUELLEMENT

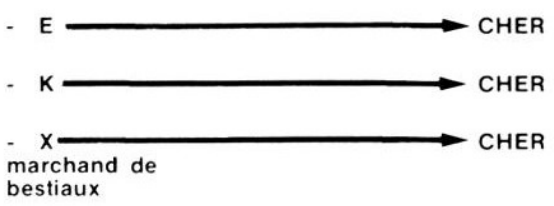

FIG. 3. - Schémas dapprovisionnement en paille. Exemples. 

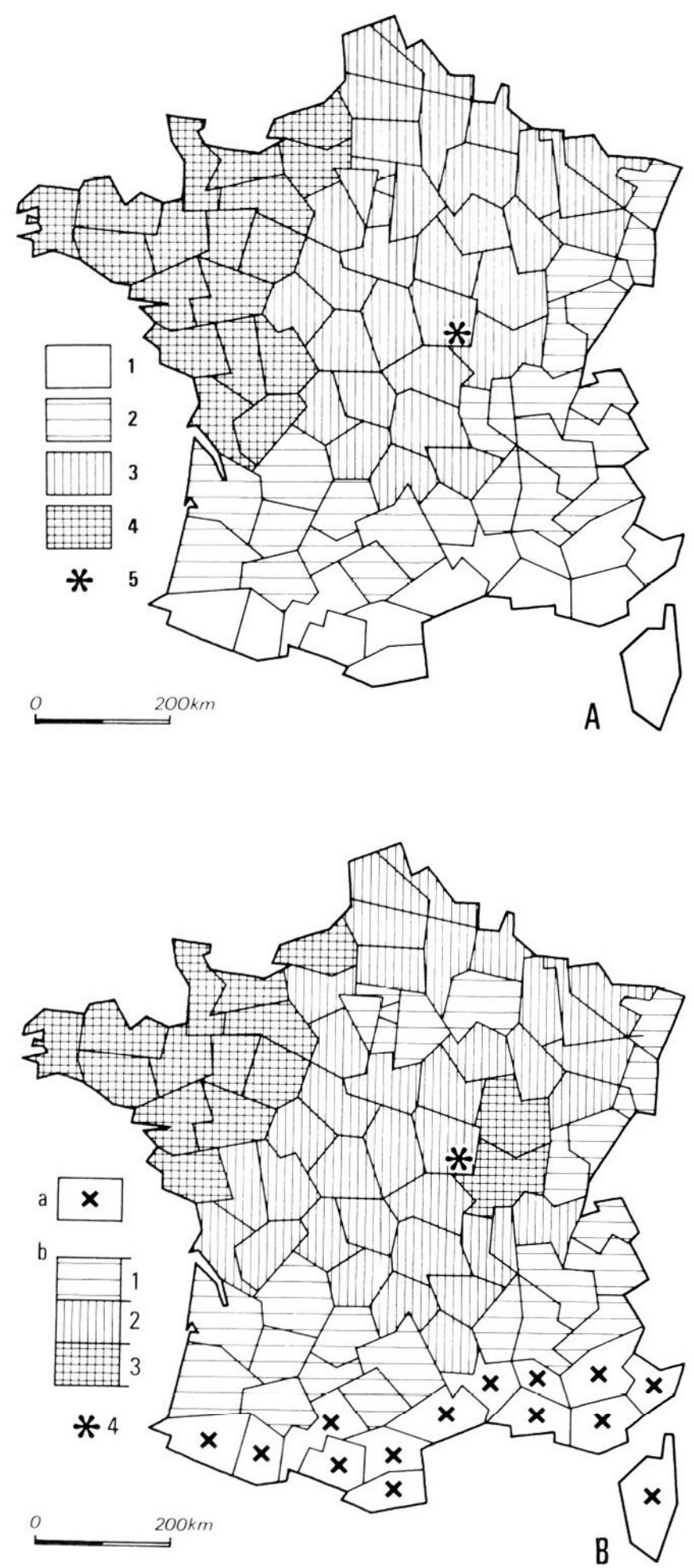

FiG. 4. - Répartition départementale des aides aux agriculteurs lors de la sécheresse de 1976.

Dans la limite de 30 UGB par exploitation individuelle. A. Aide all 25 août: 1. Pas daide. - 2. 50F/UGB. 3. $150 \mathrm{~F} / \mathrm{UGB}$. - 4. 200F/UGB. - 5. Canton de Luzy.

B. Aide all 7 octobre: a. Prise en charge des intérêts des prèts. b. 1. Entre 100 et $200 \mathrm{~F} / \mathrm{UGB}$. - 2. Entre 200 et $300 \mathrm{~F}$ UGB. - 3. Entre 300 et $380 \mathrm{~F} / \mathrm{UGB}$. - 4. Canton de Luzy. ou par petits groupes pour n'avoir de compte à rendre à personne, ne faisant confiance qu'à euxmêmes? En fait, pour comprendre les raisons qui sous-tendent des réactions totalement individuelles ou la formation de petits groupes, il aurait fallu poser des questions concernant l'ensemble des réseaux de relation, tant dans leur composante spatiale que dans la nature et l'ancienneté des liens sociaux (fig. 3).

Cette recherche de paille a été source de relations spatiales inusitées avec les régions voisines, céréalières: les achats se sont faits dans la Nièvre, dans l'Indre, et surtout dans le Cher, en Champagne berrichonne. Ces nouveaux réseaux se sont établis à partir de connaissances interpersonnelles, relations familiales ou relations d'affaires, pour les marchands de bestiaux notamment. Ces liens de complémentarité interrégionale pourraient se maintenir à la faveur d'une prise de conscience des inconvénients de la spécialisation régionale.

Par contre, aucune innovation socio-spatiale n'est apparue dans un domaine essentiel du système: la commercialisation des animaux. Dans cette période instable quant aux débouchés et quant aux prix, il n'était pas question de perturber les relations commerciales établies. Cette stabilité traduit un réflexe de sécurité lié au souci de ne pas prendre de risques en modifiant un système de clientélisme commercial très régulé, d'autant que la dépendance s'accroît au moment où l'offre est considérable. C'est ce même mécanisme qui explique le recours renforcé aux institutions en place, telle la coopérative, mais aussi le Crédit Agricole.

Ainsi, les comportements habituels ont conservé leur prééminence sur les pratiques nouvelles, à travers un jeu entre anciens et nouveaux réseaux de relation de la société locale, entre anciennes institutions et nouveaux «systèmes» de défense. Il semblerait donc que la crise n'ait pas été suffisamment grave pour susciter des solutions collectives nouvelles durables. Etait-ce que l'aléa climatique n'avait pas eu l'ampleur et la durée suffisantes pour entraîner un déséquilibre profond ou (et) que le système socio-spatial pouvait amortir le traumatisme grâce aux seules forces d'innovation de ses individus et de ses institutions?

\section{CONCLUSION}

Cet essai sur la sécheresse de $1976 \mathrm{chez}$ les éleveurs d'un canton de la Nièvre nous a permis, par le biais de l'accident climatique, de porter un regard nouveau sur les rapports homme-milieu et sur le fonctionnement d'une société agricole.

D'une part, ce moment critique a conduit à reconnaître à nouveau la valeur de l'équilibre entre le système de production et le milieu physique: une 
conception de l'exploitation fondée sur le progrès technique et la croissance économique, conjuguée avec une succession de bonnes années l'avaient fait oublier. La question s'est alors posée de savoir si la croissance continue du cheptel reproducteur sur unc surface constante pcut se fairc sans la recherche d'une augmentation au moins équivalente des ressources alimentaires autonomes. La remise en honneur des notions d'autosuffisance, de recherche d'une certaine autonomie des exploitations agricoles, comme d'ailleurs des collectivités a rejoint les idées ambiantes diffusées par une partie du mouvement écologique, en réaction contre la dépendance technique que connaissent les exploitations et les régions très spécialisées.

A l'intérieur du processus général de remodelage de l'activité agricole, le phénomène imprévu paraissait devoir être à la fois révélateur des clivages dans la société agricole et agent d'une accélération conjoncturelle des restructurations. Or notre enquête, en fait, a souligné le clivage majeur, traditionnel, entre les très grandes exploitations et toutes les autres. Mais, rien ne nous permet d'affirmer que la sécheresse ait pu creuser les inégalités entre exploitations. Tout au plus pourrait-on dire que l'écart entre les grandes exploitations et les petites et moyennes s'est maintenu. L'observation de cas de faillite aurait été éclairante : il n'en a pas existé sur le moment. Il ne faudrait pas sous-estimer pour cela les effets à terme, même indirects, qui ont pu conduire, plusieurs années après, à de graves déséquilibres, voire à des cessations d'exploitations, liées par exemple à des pénuries en crédit.

On n'en reste pas moins étonné devant les capacités de résistance des exploitations et l'éventail assez large des façons d'intégrer l'aléa. A la limite, il s'agit de la mobilisation de toutes les forces en outils, en travail, en ressources financières, non seulement de l'exploitation, mais aussi de la famille. Ce qui renvoie à la difficulté qu'il y a, dans la plupart des agricultures européennes, à cerner ce qui res- sortit au cadre familial, et ce qui ressortit à l'exploitation agricole stricto sensu.

Pour ce qui est des comportements collectifs, l'enquête a mis en évidence l'existence d'un consensus à l'intérieur de la société agricole, si limité qu'il soit; un certain esprit de corps s'est manifesté, par-delà les divisions, à propos des politiques mises en œuvre et des solutions préconisées par tous. L' «impôt sécheresse » a gêné tous les agriculteurs, parce qu'il les renvoyait dans un ghetto professionnel, face aux autres catégories de travailleurs. Mais ils auraient souhaité la taxation des prix des produits industriels nécessaires à l'agriculture et ont trouvé justifiée, voire insuffisante, la subvention accordée. Cependant, la crise n'a, semble-t-il, pas été suffisamment grave pour secréter une véritable mobilisation collective.

Au-delà des résultats de cette étude locale, il nous semble important de souligner l'intérêt que pcut avoir pour la géographie l'analyse d'un événement au moment de son occurrence, comme a pu tenter de le faire l'école de l' «histoire immédiate». Impliquant la mobilisation de toutes les techniques propres à notre discipline, y compris celles qui se rapportent à l'étude du milieu naturel, cette démarche permet de redonner vie à un fonctionnement social et spatial et d'échapper au «tableau géographique » statique et intemporel. Il est possible alors de mettre en évidence des tensions et des rythmes propres au milieu observé. La perception généralisante et extérieure des phénomènes (telle qu'ici, par exemple, les médias la transmettaient) est confrontée à la diversité du perçu et du vécu des intéressés, enquêtés «à chaud». Enfin, cette expérience peut permettre de prendre une distance par rapport à une vision mécaniste des phénomènes dont la grande généralité nous est connue, telle, par exemple, la disparition d'exploitations agricoles... Malgré toutes ses faiblesses et ses insuffisances, cet essai collectif nous paraît ouvrir ces quelques perspectives.

Manuscrit prêt en mars 1980 .

\section{BIBLIOGRAPHIE}

\section{SÉchERESSES.}

\section{a. Sécheresse 1976 en France.}

A.P.L. Paysans (1977), "Sécheresse 1976. Une affaire politique ». N" 174-175, juillet, non paginé.

ByÉ P., PERNet F. (1977), La sécheresse 1976. Réflexion sur la position de l'agriculture dans léconomie. Grenoble. IHRA-IREP.

B.I.M.A. (1977). «La sécheresse du printemps et de l'été 1976 ». Ministère de l'Agriculture, Bulletin n" 324-325.

Bt.Axchet G. (1977). "La sécheresse 1976 dans la région Rhône-Alpes». Revue de Géographie de Lyon. 2, p. $99-115$
Bret F. (1977), «A propos de la sécheresse. Réfllexion sur les incendies de végétation entre Rhône et Loire». Revue de Géographie de Lyon, 2, p. 153-156.

Bкоснгт P. (1976), Aspects climatologiques de la sécheresse 1976. Météorologie nationale, 6 cartes.

Charre J. (1977), "A propos de la sécheresse». Revue de Géographie de Lyon, 2, p. 215-226.

Chaval C. (1976). «La sécheresse à la fois un révélateur et un alibi de la crise \%. Economie et politique, 267, p. 44-51.

Clerc F. (1976). "La sécheresse aura été plus que la sécheresse 》. Paysans, 120, p. 4.5-52.

Dimensions économiques de la Bourgogne (1976). "Premier bilan de la sécheresse», 8, octobre, p. 3-14. 
Equipe Analyse des espaces ruraux (1979), La sécheresse de 1976 dans le canton de Luzy. Paris, Laboratoire de Géographie Humaine, CNRS, 105 p., cartes + annexes.

Gardflle C., Gardelle F. (1977), «Sécheresse et inalpage en Savoie ». Revue de géographie alpine, 2, p. 203-207.

Gazelle F. (1977), «La sécheresse 1976 en Aquitaine orientale et dans le Sud du Massif Central». Revue de Géographie des Pyrénées et du Sud-Ouest, 3, p. 245-267.

Georges B. (1976), «La sécheresse en France, ses conséquences pour l'agriculture et l'ensemble de l'économie française ». Positions, Etudes et documents pour l'action, 196, décembre, $8 \mathrm{p}$.

Gonin P. (1977), La sécheresse 1976, phénomène de conjoncture. Essai de caractérisation de ses effets dans le processus de restructuration de l'agriculture. Mémoire E.H.E.S.S., 240 p. + annexes.

Lecarpentier C. (1976), «Essai provisoire de caractérisation de la sécheresse de 1976 par l'établissement des bilans hydriques et leur confrontation avec les résultats d'études fréquentielles antérieures». Bulletin Technique d'Information, Ministèrc de l'Agriculture.

Le Monde (1976), «La sécheresse en France». Dossiers et documents $\mathrm{n}$ " $35,4 \mathrm{p}$.

Mfynier A., Macé G. (1977), «Chronique géographique de la Bretagne et du Bas-Maine. La sécheresse 19751976 ». Norois, 94, p. 297-298.

Monnier J. (1977), «Aspects et fréquence de la sécheresse en Bretagne ». Revue de Géographie de Lyon, 2, p. 197-213.

Reboul C. (1976), «Sécheresse, humus et économie marchande ». Document de Travail INRA. Economie et sociologie rurale. Paris, décembre, 33 p.

Suffert G. (1976), «La sécheresse, ses phases, ses suites, la fragilité de l'unité nationale ». Paysans, 120, p. 37-45.

Vivian H. (1977), «L'hydrologie nord-alpine et la sécheresse de 1976 ». Revue de Géographie de Lyon, 2, p. 117-151.

Vorron H., JaIl M., Loup J., Martin M. (1977), «La sécheresse dans les Alpes du Nord en $1976 »$. Revue de Géographie de Lyon, 3, p. 241-256.

\section{b. Autres sécheresses.}

PÉchoux P.Y. (1977), «La grande sécheresse 1973 à Chypre». Revue de Géographie de Lyon, 2, p. 197-213.

La sécheresse au Sahel pose des problèmes de nature et d'échelle tout à fait différentes. Une importance bibliographie lui est consacrée. Nous n'en donnons ici que quelques orientations :

Beudot F. $(1977,1978,1979)$. Eléments de bibliographie sur la sécheresse au Sahel. Mise à jour, n" 1, 93 p.; Mise à jour n" 2, 149 p.; mise à jour, n" 3, avril 1979, $131 \mathrm{p}$.

Comité d'Information Sahel, (1975), Qui se nourrit de la famine en Afrique? Le dossier politique de la faim au Sahel. Paris, Maspéro, 197 p.

Copans J. (éd.) (1977), Sécheresse et famine au Sahel. Paris, Maspéro, 2 vol., 155 p. et 138 p.

Dresch J. (1977), «Géographie et Sahel ». Hérodote, 6, p. $54-71$.

Joyce S.J., Beudot F. (1976), Eléments de bibliographie sur la sécheresse au Sahel. Paris, OCDE, $131 \mathrm{p}$.
Notes et Etudes Documentaires (1975), «La sécheresse en zone sahélienne. Causes. Conséquences. Etudes des mesures à prendre ». Paris, La Documentation française, n" 4216-4217, 75 p.

Sahel documentatie (1977), Uitgave koninklijk, Instituut voor de Tropen, Amsterdam n" 1, juin-sept.

Travaux de l'Institut de Géographie de Reims (1979), «La désertification », 39-40, $127 \mathrm{p}$.

Travaux et documents de géographie tropicale (1977), «Stratégies pastorales et agricoles des Sahéliens devant la sécheresse 1969-1974. Elevage et contacts entre pasteurs et agriculteurs». Bordeaux, CEGET, $281 \mathrm{p}$.

\section{Crises, catastrophes naturelles.}

a. Recherches françaises.

Bontron J.C., Plet F., Rey V., Robic M.C. (1978), «Cadres et modes de vie en milieu rural. Réflexions sur les agriculteurs dans les marges sud-est du Bassin parisien ». Communication aux Journées de Géographie rurale, Aix-en-Provence, $13 \mathrm{p}$.

Brunet R. (1968), Les phénomènes de discontinuité en géographie. Paris, CNRS, Coll. Mémoires et Documents, $119 \mathrm{p}$.

Baunet R. (1974), «Espace, perception et comportement 》. L'Espace géographique, 3, p. 189-204.

Claval P. (1974), «La géographie et la perception de l'espace ». L'Espace géographique, 3, p. 179-187.

JAMmes A.M., REY V. (1974), «Les géographes ruraux et le milieu naturel». Approche géographique des exploitions agricoles, Cahier n“ 2, LA 142, p. 165-172.

Guigou J.L., Lebailly M., Pierzo D., Uzan L.J. (1979), Les nouveaux espaces de solidarité. Etude préliminaire sur lu pertinence du concept. IRU Enviromement, Université Paris Val-de-Marne, $156 \mathrm{p}$

PÉGuy C.P. (1979), «Ordre et désordre des climats». L'Espace géographique, 1, p. 5-14.

Poncet J. (1975), «L'intérêt du concept de cataclysme». LEspace géographique, 1, p. 65-72.

UZAN L. (1978), «Pratiques sociales, pratiques spatiales en milieu rural : quels schémas, pour quels espaces? » Table ronde du 30 mai 1978. Pratiques sociales et pratiques spatiales en milieu rural. Exposés de quelques recherches. IRU Environnement, Université Paris Valde-Marne.

b. Recherches étrangères.

Burton I., Kates R.W., White G.E. (1978), The environment as hazard. New York, Oxford University Press, $240 \mathrm{p}$.

Geipel R. (1977), «Friaul, Sozialgeographische Aspekte einer Erdbebenkatastrophe». Münchener geographische Hefte, 40 Kallmünz/Regensburg. Verlag Michaël Lassleten, $212 \mathrm{p}$.

GeIPEL R. (1978), «La géographie de la perception en Allemagne fédérale ». L'Espace géographique, 3, p. 195-198.

Lowenthal D. (ed.) (1967), «Environmental perception and behavior ». Chicago, University of Chicago, Department of Geography, Research paper n" 109, 88 p., dont KATES R.W. "The perception of storm hazard on the shores of mega'opolis», p. 60-74. 
Parker D.J., Harding D.M. (1979), « Natural hazard evaluation, perception and adjustment ». Geography, 4, p. 307-316.

Pecsi M., Probald F. (ed.) (1974), Man and environnement. Budapest, Akademiai kiadó, 245 p. dont, WhITE G.F., "Comparative ficld observations on natural hazards», p. 73-79; White A.U., «Toward a global summary of natural hazard», p. 81-85.
SaARINEN T.F. (1966), "Perception of the drought hazard on the Great Plains». Chicago, University of Chicago, Department of Geography, Research paper n" 106, XII, 187 p.

White G.F. (ed.) (1974), Natural hazards. Local, national, global. New York, Oxford. University Press, 288 p.

Signalons la parution récente du n* 24-1982 d'Hérodote sur le thème: terres à hauts risques.

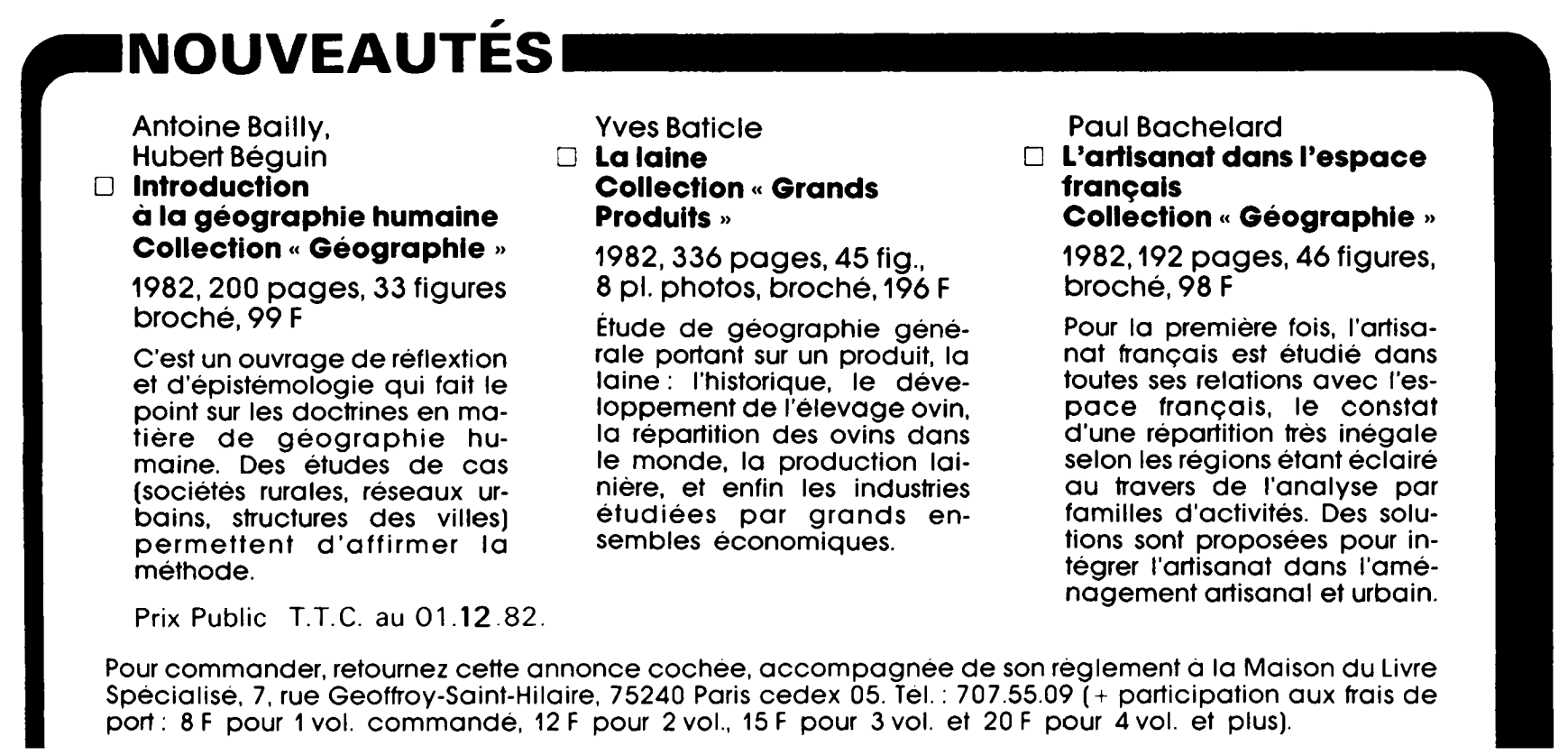

